\title{
Patellar maltracking: an update on the diagnosis and treatment strategies
}

\author{
Zaid Jibri ${ }^{1,2^{*}}$, Paul Jamieson ${ }^{2,3}$, Kawan S. Rakhra ${ }^{1,2}$, Marcos L. Sampaio ${ }^{1,2}$ and Geoffrey Dervin ${ }^{2,3}$
}

\begin{abstract}
Patellar maltracking occurs as a result of an imbalance in the dynamic relationship between the patella and trochlea. This is often secondary to an underlying structural abnormality. The clinical evaluation can provide useful clues for the presence of such entity; however, the diagnosis can often be challenging especially in the absence of a documented history of patellar dislocation. Imaging, particularly MRI, can detect subtle features that could lead to the diagnosis, probably even more importantly when there is no clear history of patellar dislocation or before its development. This can provide a road map for formulating a treatment strategy that would be primarily aimed at stabilizing the patellofemoral joint to halt or slow the progression of articular cartilage loss. The purpose of this article is to discuss the clinical and radiologic evaluation of patellar maltracking providing an update on the crosssectional imaging assessment and also a synopsis of the management options.
\end{abstract}

Keywords: Patellar maltracking, Patellar instability, Anterior knee pain, Patellofemoral osteoarthritis

\section{Key points}

- The clinical evaluation of patellar maltracking is often challenging

- Imaging can detect subtle features that could lead to early diagnosis

- Imaging can detect predisposing factors for patellar maltracking and associated structural changes

- Management decisions are made on individual basis with imaging playing a vital role

\section{Background}

Patellar tracking refers to the dynamic relationship between the patella and trochlea during knee motion [1]. Patellar maltracking occurs as a result of imbalance of this relationship often secondary to anatomic morphologic abnormality. Usually, young individuals, particularly women, suffer the consequences of this disorder [2]. It is a recognized cause of anterior pain and in extreme cases presents as acute and often recurrent patellar dislocation, which is usually transient. Early diagnosis

\footnotetext{
* Correspondence: zjibri@toh.ca

'Department of Medical Imaging, The Ottawa Hospital, 501 Smyth Road,

Ottawa, ON K1H 8L6, Canada

${ }^{2}$ Faculty of Medicine, University of Ottawa, 451 Smyth Road, Ottawa, ON K1H 8M5, Canada

Full list of author information is available at the end of the article
}

is essential, as chronic maltracking will lead to patellofemoral cartilage damage and osteoarthritis [3]. Imaging, particularly MRI, plays a vital role in the assessment of patellar maltracking. It can not only detect any underlying morphological risk factors but also look for structural damage associated with maltracking including patellofemoral articular cartilage loss, osteochondral defects, or damage to the medial patellar stabilizers $[4,5]$. The purpose of this article is to discuss the evaluation of patellar maltracking providing an update on the imaging assessment and also a synopsis on the management options.

\section{Biomechanics of the patellofemoral joint}

The knee is a complex joint with separate tibio-femoral and the patellofemoral articulations. Understanding the biomechanics of these joints is essential to investigating and appropriately treating patellofemoral joint pathology. The patellofemoral joint has two primary functions; firstly, it acts as an anatomic pulley to provide mechanical advantage for the extensor mechanism and, secondly, to reduce friction between the extensor mechanism and the femur. The patella itself is shaped as an inverted triangle and is embedded in the quadriceps tendon, making it the largest sesamoid bone in the body [6]. Distally, it attaches to the tibial tubercle via the patellar tendon. The posterior articulating surface of the 
patella is composed of two facets, a medial and lateral facet, separated by a vertical ridge, and in $30 \%$ of the population, there is a third facet, the odd facet, most medially. The patella articulates with the trochlear groove of the anterior femur, which has corresponding lateral and medial patellar articular surfaces [6]. The lateral trochlear articular surface is usually more prominent than its medial portion. As the knee joint ranges from extension to flexion, the articular surface area of the patella is in contact with the femur changes. In full extension, the patella has little to no contact with the trochlear groove and, therefore, is in a position of higher risk for instability. From 10 to $20^{\circ}$ of flexion, the patella engages the trochlear groove with the contact area being the inferior most portion of the medial and lateral facets. As the knee progresses through greater flexion, the contact surface becomes more proximal on the patella. It is not until beyond $90^{\circ}$ of flexion that the odd facet engages the medial femoral condyle and plays a role in load sharing along with lateral facet $[6,7]$. Additionally, in this degree of flexion, the quadriceps tendon itself engages the proximal trochlear groove and participates in force distribution [8-10].

The patella has 4 different planes of motion: flexionextension, medial-lateral rotation, medial-lateral patellar tilt, and medial-lateral patellar shift. The stability of the patella is dependent on both osseous anatomy and the integrity of longitudinal and transverse soft tissue stabilizers. The transverse stabilizers include the medial and lateral retinaculum, the vastus medialis and lateralis muscles, the ilio-tibial band, and the medial patellofemoral ligament (MPFL). The longitudinal stabilizer is the extensor mechanism itself, which is comprised of the quadriceps tendon proximally and the patellar tendon distally. The anatomic relationship between the resultant force from the quadriceps and the line of pull of the patellar tendon is termed the Q angle and is normally 10$15^{\circ}$ of valgus [11]. This results in a slightly superolateral direction of pull on the patella by the quadriceps. Correspondingly, the patella must shift slightly medially during early flexion to engage the trochlear groove.

\section{Clinical presentation and initial evaluation}

The most obvious presentation of patellar maltracking is that of the first time lateral patellar instability or recurrent instability thereafter. Less commonly, patients can also present after chronic patellar instability secondary to generalized ligamentous laxity with or without anterior knee pain. Although varied in presentation, successful management of all patients relies on thorough history taking, physical examination of the entire lower extremity, and appropriate imaging. The clinical evaluation can be more challenging in the absence of a dislocation history, and in this scenario, imaging can have a critical role.

Acute traumatic instability most commonly occurs in young athletes in their second and third decade at an incidence rate of 29 per 100,000. Some controversy exists regarding whether female gender is a definite risk factor for patellar instability with certain studies identifying a $33 \%$ increased likelihood of first-time dislocation as well as three times high re-dislocation rates than males, whereas others have found roughly equal rates [2, 12-14]. The mechanism is commonly a non-contact twisting injury of the lower extremity with the knee extended and external rotation of the foot and is perceived as the knee "giving way." The patella will often self-reduce by reflexic contraction of the quadriceps muscles. Less commonly, a direct laterally or medially orientated blow to the patella can precipitate dislocation. Traumatic dislocations are commonly associated with other injuries including that of the MPFL, meniscal pathology, and osteochondral fractures of the femur or patella $[15,16]$.

Between 15 and $45 \%$ of patients will develop recurrent patellar instability after acute dislocation, which is both functionally limiting and painful [17-20]. Risk factors for recurrent instability include female sex, family history of patellar instability, and various anatomic risk factors such as patella alta, increased femoral anteversion, external tibial rotation, genu valgum, trochlear dysplasia, increased tibial tubercle-trochlear groove (TT-TG) distance, and patellar tilt [13, 21-23]. These prevailing anatomic indices feature prominently into the probability of recurrence, and understanding their variability and pathophysiology is critical to successful management of these patients.

A focused history of the mechanism, number, and circumstances of instability to date is essential. A generalized physical examination assessing ligamentous laxity and rotational profile of the lower extremity is critical. A thorough examination of the knee is then performed including presence of effusion, localization of pain, assessment of patellar translation, patellar apprehension, presence of a J sign (visual lateralization of the patella as it disengages from the trochlea when extending the knee), and a measurement of the $\mathrm{Q}$ angle along with ligamentous and meniscal testing. Distal neurovascular examination also needs to be performed [16].

\section{Imaging techniques}

Imaging assessment can start with the radiograph including anteroposterior and lateral views of the knee and skyline view of the patella. The radiograph can be helpful in the acute presentation in detecting fractures in the setting of lateral (often transient) patellar dislocation. However, it lacks sensitivity with $40 \%$ of sizable osteochondral lesions being missed on initial presentation after patellar 
dislocation [16]. The radiograph can also be useful in detecting osseous morphologic features associated with patellar maltracking such as patella alta and trochlear dysplasia [24, 25]. MRI and CT are superior modalities in looking for predisposing factors associated with patellar maltracking [26-28]. In this section, we will emphasize the role of MRI and discuss how CT can also have value when assessing patellar maltracking.

\section{Magnetic resonance imaging features}

Magnetic resonance imaging (MRI) is a vital tool in evaluating the potential cause(s) of anterior knee pain due to the complexity of the structure and biomechanics of the knee. Various parameters can be used in assessing and predicting the presence of patellar maltracking. These parameters can be evaluated using dynamic MRI [29]. However, the use of this method is not widespread. On the other hand, there are static MRI measurements that are routinely used as indicators of patellofemoral alignment during knee movement [30, 31]. The main morphological features associated with patellar maltracking are trochlear dysplasia, lateralization of the tibial tuberosity, patella alta, and lateral patellar tilt. MRI is the imaging modality of choice in the assessment of patellar maltracking, as a virtue of what it can reveal (Table 1).

\section{Trochlear dysplasia}

It is a geometric abnormality of the trochlear groove that affects its shape and depth mainly at its superior part, which can result in abnormal tracking of the patella along the trochlea. It is a major factor in patellar instability and was shown to be present in $85 \%$ of these patients [21]. There are a number of MRI features of trochlear dysplasia including reduction in the trochlear depth, lateral trochlear inclination, and facet asymmetry. These are evaluated on most cranial axial image showing cartilage, approximately $3 \mathrm{~cm}$ above the joint line. The trochlear depth is calculated by measuring the mean of the maximum anteroposterior (AP) distance of the medial and lateral femoral condyles minus the distance between the deepest point of the trochlear groove and the line paralleling the posterior femoral condyles surfaces (Fig. 1). Less than 3-mm trochlear depth is indicative of trochlear dysplasia [24]. Lateral trochlear inclination is another quantitative method to diagnose trochlear dysplasia. It is the angle between a line tangential to the subchondral bone of the posterior aspect of the femoral condyles and a line along the lateral trochlear facet subchondral bone (Fig. 2). An inclination angle of less than $11^{\circ}$ indicates trochlear dysplasia [32]. Facet asymmetry is determined by calculating the percentage of the medial to the lateral femoral facet length (Fig. 3). Asymmetry of $<40 \%$ suggests trochlear dysplasia [24].

Dejour et al. provided a morphologic classification system for trochlear dysplasia describing four types [26-28]. In type $\mathrm{A}$, the trochlear preserves its concave shape but has shallow trochlear groove; type $\mathrm{B}$ is flattened or convex trochlea; in type $\mathrm{C}$, the medial facet is hypoplastic (facet asymmetry) with high lateral facet, resulting in flattened joint surface in an oblique plane; and type D shows a "cliff pattern" with type $C$ features and a vertical link between the medial and lateral facets.

\section{Patella alta}

Patella alta is related to a long patellar tendon and is considered a major factor associated with reduced contact

Table $1 \mathrm{MRI}$ checklist for the assessment of patellar maltracking

\begin{tabular}{|c|c|c|}
\hline $\begin{array}{l}\text { Patellar maltracking- } \\
\text { associated feature }\end{array}$ & Methods of assessment (amongst others) & Significance \\
\hline Trochlear dysplasia & $\begin{array}{l}\text { Trochlear depth (Fig. 1), lateral trochlear inclination (Fig. 2), } \\
\text { trochlear facet asymmetry (Fig. 3) (evaluated on most cranial } \\
\text { axial image showing cartilage, approximately } 3 \mathrm{~cm} \text { above } \\
\text { the joint line) }\end{array}$ & $\begin{array}{l}\text { Geometric abnormality of the trochlear groove that can result in } \\
\text { abnormal tracking of the patella along the trochlea }\end{array}$ \\
\hline Patella alta & $\begin{array}{l}\text { Insall-Salvati index (Fig. 4) } \\
\text { Caton-Deschamps index (Fig. 4) }\end{array}$ & $\begin{array}{l}\text { Relates to a long patellar tendon. In order for the patella to } \\
\text { engage with the trochlea, a higher degree of flexion than } \\
\text { normal is needed }\end{array}$ \\
\hline $\begin{array}{l}\text { Lateralization of the } \\
\text { tibial tuberosity }\end{array}$ & Tibial tubercle-trochlear groove distance (TT-TG) (Fig. 5) & $\begin{array}{l}\text { High TT-TG would exert lateral pressure on the patella during } \\
\text { extension, and if not counteracted by vastus medialis contrac- } \\
\text { tion, it may predispose to patellar subluxation }\end{array}$ \\
\hline Lateral patellar tilt & $\begin{array}{l}\text { Patellar tilt angle (Fig. 6) } \\
\text { Patellofemoral angle }\end{array}$ & $\begin{array}{l}\text { Sensitive marker for patellar instability present in significant } \\
\text { proportion of patients }\end{array}$ \\
\hline $\begin{array}{l}\text { Hoffa's fat pad } \\
\text { impingement }\end{array}$ & Edema at the superolateral aspect of Hoffa's fat pad on MRI & $\begin{array}{l}\text { Significant association with several patellar maltracking } \\
\text { indicators }\end{array}$ \\
\hline $\begin{array}{l}\text { MPFL and medial } \\
\text { patellar retinacular } \\
\text { injury }\end{array}$ & Best evaluated on the axial fluid sensitive MRI sequence & Present in the majority of patellar dislocation cases \\
\hline $\begin{array}{l}\text { Chondral and } \\
\text { osteochondral } \\
\text { damage }\end{array}$ & $\begin{array}{l}\text { MRI can show discrete osteochondral defect or various } \\
\text { degrees of patellofemoral cartilage loss }\end{array}$ & $\begin{array}{l}\text { Patellar maltracking is significant risk factor patellofemoral } \\
\text { osteoarthritis. Patellar dislocation can result in discrete } \\
\text { osteochondral defects at the patella or lateral femoral condyle }\end{array}$ \\
\hline
\end{tabular}




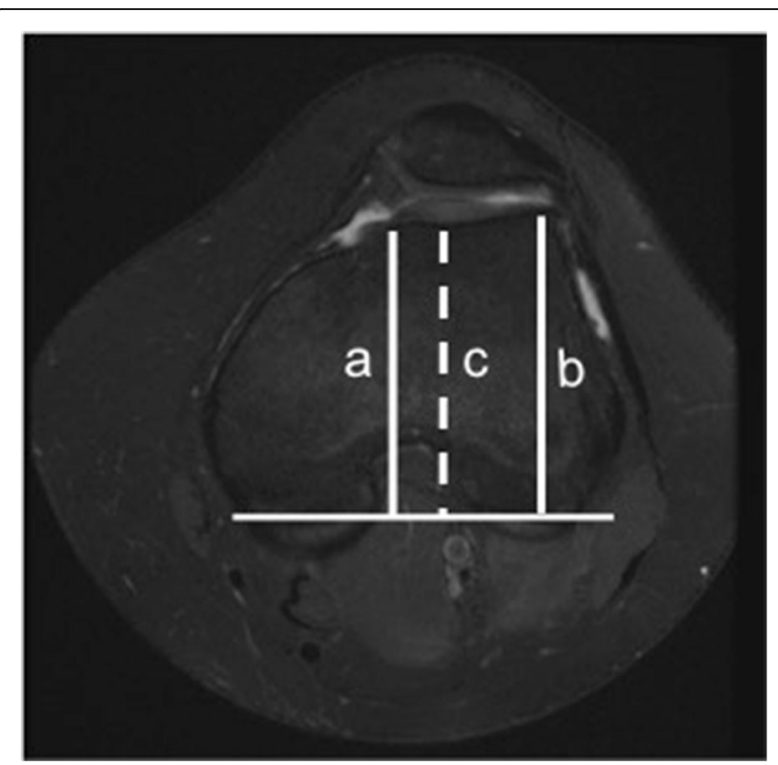

Fig. 1 Trochlear depth assessment. Axial PDFS left knee MR image, demonstrating the method used for the measurement of trochlear depth. First, a line is drawn paralleling the posterior femoral condyles surfaces. Perpendicular to this baseline, trochlear depth is calculated by measuring the mean of the maximum AP distance of the medial (a) and lateral (b) femoral condyles minus the distance between the deepest point of the trochlear groove and the line paralleling the posterior condylar surfaces $(\mathbf{c})$. Trochlear depth $=[(a+b) / 2]-c$

area at the patellofemoral joint and a major contributor to patellar instability [33]. In order for the patella to engage with the femoral trochlea, a higher degree of flexion than normal is needed. Several methods have been used to assess patella alta. A commonly used one is the Insall-Salvati ratio of patellar tendon length: patellar length. This is measured on the sagittal MRI images at the point where the patella is at its greatest length. It is a ratio between the patellar tendon length (along the inner surface of the tendon) and the diagonal patellar height [27]. A ratio of $>1.3$ is considered indicative of patella alta [34] (Fig. 4). Another method is the Caton-Deschamps index. This is the ratio between a line measured between the inferior margin of the patellar articular surface and the anterior aspect of the tibial plateau and the greatest length of the patellar articular surface. A ratio equal or more than 1.2 indicates patella alta [35] (Fig. 4). A newer method to assess for patella alta is the patellotrochlear index (PTI), which is measured in the midsagital MRI as the ratio of the length of trochlear cartilage engaged with the patella to the patellar cartilage length [36]. PTI of less than $12.5 \%$ suggests the presence of patella alta. Each of the mentioned assessment methods of patella alta has its own advantages and limitations. As an example, although the Insall-Salvati ratio is one of the most commonly used methods and does not depend on the degree of knee flexion, it is affected by the patellar shape particularly its inferior point and measurement does not change after tibial tubercle distalization procedure [25]. On the other hand, the PTI is significantly altered with knee flexion [37].

\section{Tibial tubercle-trochlear groove distance}

An increased tibial tubercle-trochlear groove (TT-TG) indicates a lateralized tibial tuberosity, or a medialized trochlear groove [38]. TT-TG is a reflection of the clinically measured $\mathrm{Q}$ angle. A high $\mathrm{Q}$ angle or TT-TG would exert a lateral pressure on the patella during knee extension, and if this is not counteracted by vastus medialis muscle contraction, it can predispose to lateral patellar subluxation and instability $[39,40]$. There is a degree of variability in the literature about what is considered an abnormally high TT-TG. TT-TG distance of more than $20 \mathrm{~mm}$ is believed to be nearly always associated with patellar instability [27].

The TT-TG is evaluated by measuring the distance between the most anterior point of the tibial tuberosity and the deepest point of the trochlear groove using two

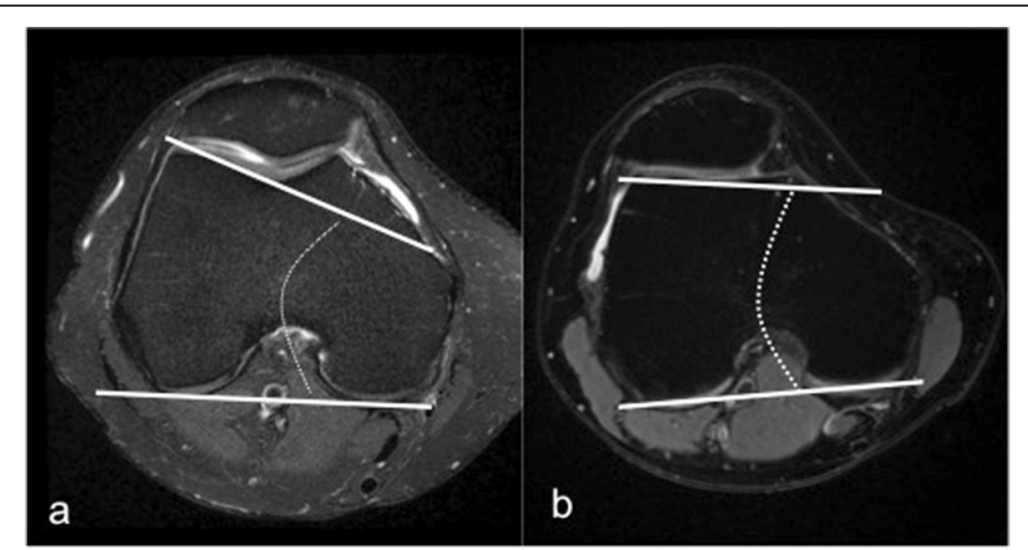

Fig. 2 Lateral trochlear inclination measurement on axial MRI. It is the angle between a line tangential to the subchondral bone of the posterior aspect of the femoral condyles and a line along the lateral trochlear facet. a Normal trochlea. b Trochlear dysplasia ( $9^{\circ}$ inclination) 


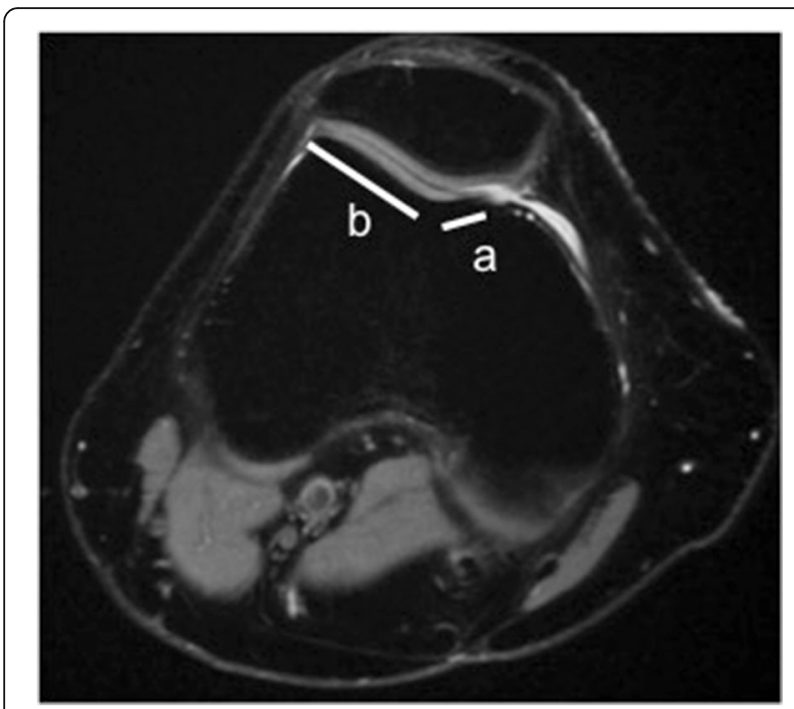

Fig. 3 Facet asymmetry assessment for trochlear dysplasia on axial MRI. It is the percentage of the medial (a) to the lateral (b) trochlear facet length $(\mathrm{a} / \mathrm{b} \times 100 \%)$

lines drawn perpendicular to the tangent to the posterior borders of the femoral condyles [31] (Fig. 5). TT-TG assessment has its own limitations. The TT-TG distance can be influenced by the degree of knee flexion (reduces with flexion), and it is also smaller upon weight bearing [41]. It can be difficult to determine the deepest part of the trochlear groove when assessing the TT-TTG in the presence of trochlear dysplasia; therefore, an alternative method for assessing tibial tubercle position was proposed measuring the distance in reference to the posterior cruciate ligament and not to the trochlea (tibial tubercle-posterior cruciate ligament distance [TT-PCL]), with proposed pathologic threshold of $21 \mathrm{~mm}[42,43]$. More recently, the TT-TG index was developed, which takes knee size into account by assessing the proximaldistal distance between the entrance of the chondral trochlear groove (TE) and the tibial tuberosity (TT). The TT-TG index is the TTTG/TT-TE ratio [44].

\section{Lateral patellar tilt}

Lateral patellar tilt is a sensitive marker for patellar instability [45]. It may occur without patellar lateralization. In a series of 474 patients with anterior knee pain, patellar tilt or subluxation was present in $40 \%$ of the cases on axial MRI [46]. The degree of patellar tilt can be evaluated by measuring the patella tilt angle, which is the angle between the posterior condylar line and the maximal patella width line [47] (Fig. 6). Patellar tilt can also be assessed using the patellofemoral angle (PFA). PFA is the angle between a line drawn along the bony lateral patellar facet and another line along the anterior aspect of the femoral condyles. It is measured at the mid-point of the patella on the axial slices [48]. PFA of $0^{\circ}$ or if it opens medially (negative value) is considered abnormal indicating lateral patellar tilt $[27,48]$.

\section{Structural changes associated with patellar maltracking Injuries to the medial patellar stabilizers}

It has been shown that damage to the medial patellar stabilizers including medial patellar retinaculum and the medial patellofemoral ligament (MPFL) injuries are prevalent in $70-100 \%$ of cases of lateral patellar dislocation [5, 27, 49-51]. The medial patellar retinaculum and MPFL are best seen on MRI on the axial fluid-sensitive sequences. These two structures blend with each other and are difficult to separate on imaging. The MPFL is attached to the region of the adductor tubercle or medial femoral epicondyle extending deep to the vastus medialis obliquus (VMO) and attaching to the superior two thirds of the patella [52]. In one MRI study, $76 \%$ of cases of prior lateral patellar dislocation showed medial retinacular injury at its patellar insertion and 30\% at its midsubstance, and injury of the femoral origin of the MPFL

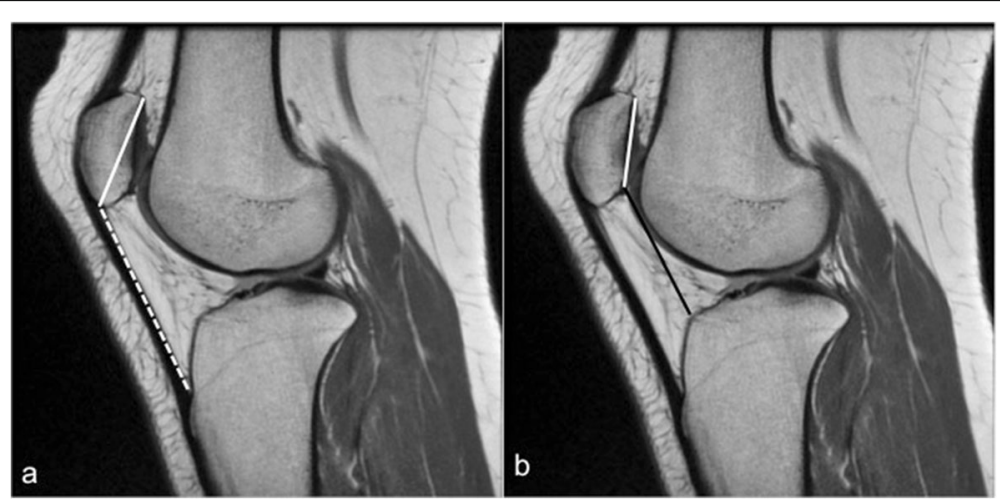

Fig. 4 Patella alta assessment. a Sagittal PD knee MRI showing the method of assessing the Insall-Salvati index, calculated as the ratio of the patellar tendon length at its inner aspect (white dashed line) to the greatest diagonal length of the patella (white line). b Patellar alta evaluation using the Caton-Deschamps index, which is the ratio between a line measured between the inferior margin of the patellar articular surface and the anterior aspect of the tibial plateau (black line) and the greatest length of the patellar articular surface (white line) 


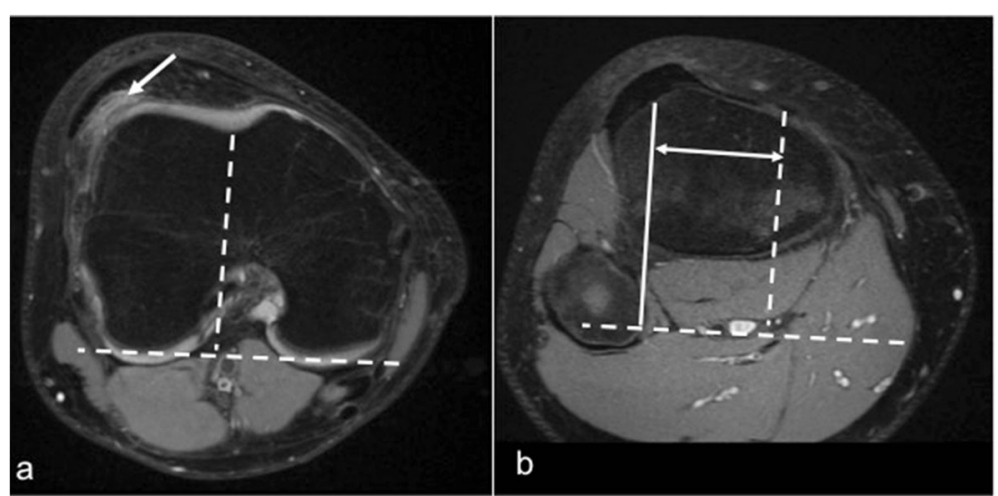

Fig. 5 Tibial tubercle-trochlear groove distance (TT-TG) assessment. a Axial PDFS right knee MR image at the level of the trochlear groove. $\mathbf{b}$ Axial MRI at the level of the tibial tuberosity. TTG is the distance between the solid and the dashed lines in (b). Note the edema in the superolateral aspect of Hoffa's fat pad (arrow)

was identified in $49 \%$ of the cases [49]. Both MRI and ultrasound are accurate imaging modalities in the detection of MPFL injuries [5, 50, 51]. The patellar retinaculum and the MPFL are seen on MRI as well-defined low-signal-intensity bands. The MPFL is best seen on axial MRI on the slice just distal to the VMO. On T2weighted MR images, sprain is depicted as thickening of retinaculum with signal intensity signifying edema and hemorrhage (Fig. 7). Complete disruption and avulsion are seen as discontinuity of ligament fibers with associated edema [50]. It has been shown that ossification in the medial patellar stabilizers correlates with prior injury to these structures [53].

\section{Superolateral Hoffa's fat pad impingement}

Infrapatellar (Hoffa's) fat pad impingement is recognized as a cause of anterior knee pain. Chronic fat impingement can result in chronic inflammation and fat pad hypertrophy. On MRI, impingement is usually manifested as high signal intensity within the superolateral aspect of the infrapatellar fat pad on fluid-sensitive sequences (edema) (Fig. 5). It has been shown that there is an association between edema at the superolateral aspect of Hoffa's fat pad and a number of patellar maltracking parameters $[30,54,55]$. It has been suggested that fat impingement occurs between the lateral femoral condyle and the posterior aspect of the patellar tendon [54]. Identifying edema at the superolateral aspect of Hoffa's fat pad on MRI should prompt the reporting radiologist to look for features of patellar maltracking. Although edema can be seen in other peripatellar fad pads on MRI, there is no clear association between patellar maltracking and prefemoral fat pad edema or with that at the suprapatellar fat pad [56].

\section{Chondral and osteochondral injuries}

An imbalance of forces acting on the patellofemoral joint due to abnormal bony geometry or altered function of the active and passive soft tissue restraints may result in abnormalities of alignment and tracking of the patella. Stress and shear forces that follow can result in cartilage

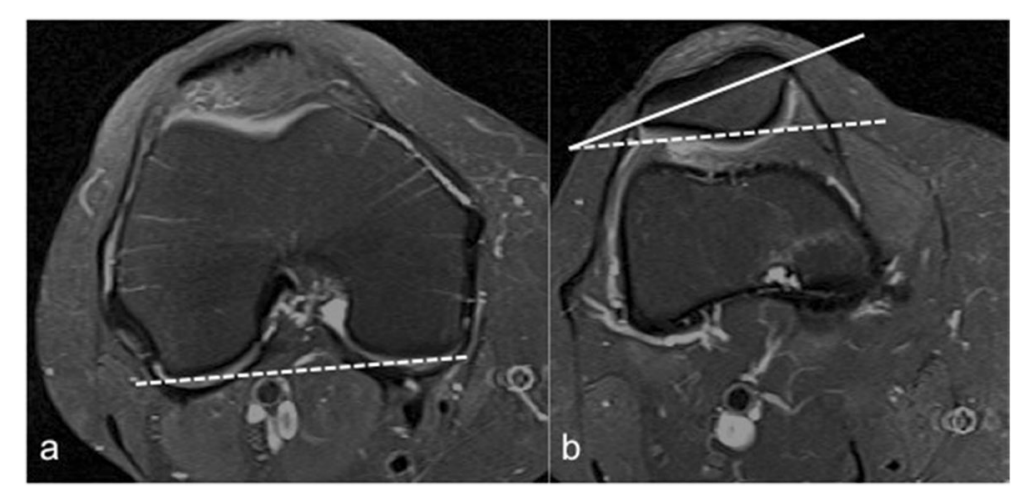

Fig. 6 Patellar tilt assessment. a The posterior condylar line is drawn on the slice where the posterior femoral condyles are largest (dashed line). $\mathbf{b}$ The patella tilt angle is measured between the posterior condylar line (dashed line) and the maximal patella width (solid line). 


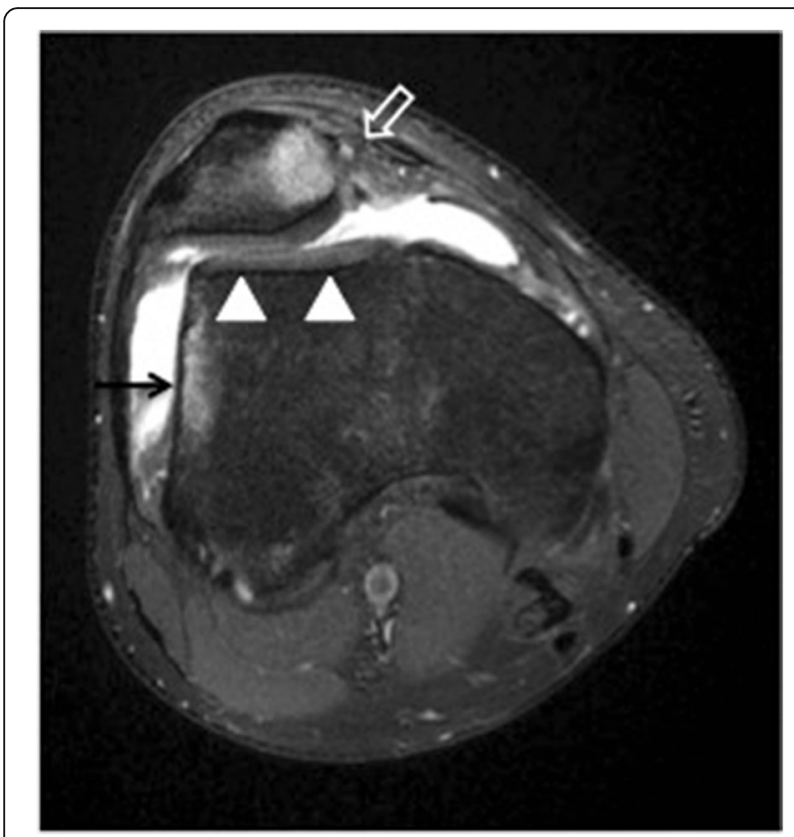

Fig. 7 Transient lateral patellar dislocation. Axial PDFS MR image showing MPFL disruption (open arrow) and trochlear dysplasia (arrowheads). There is edema of the medial patella and of the lateral femoral condyle (arrow), consistent with bone contusion due to recent lateral patellar dislocation

damage and the development and evolution of osteoarthritis [57].

MRI, given its superior soft tissue contrast and multiplanar capability, has emerged as the modality of choice in evaluating articular cartilage abnormalities. It has proven to be both sensitive and specific in the detection of hyaline cartilage abnormalities [4].

An association has been demonstrated between patellofemoral cartilage damage and patellar maltracking. A study has found that the femoral groove tends to be shallower in osteoarthritis patients compared to those with normal cartilage, regardless of age. Significantly greater lateral patellar displacement and tilt was found in osteoarthritis patients compared to a control group [3]. Another study noted an association between abnormal trochlear morphology and high-grade patellofemoral cartilage damage [58]. It was shown that certain features of patellar maltracking (increased sulcus angle, lateral patellar tilt, and a higher patellar tendon to patellar length ratio) are associated with cartilage loss and bone marrow lesions [59].

On the other hand, frank patellar dislocation is a significant risk factor in the development of patellofemoral osteoarthritis with an incidence of $49 \%$ at 25 years after the patellar dislocation incident in comparison with $8 \%$ in a control group without a dislocation history [60]. Lateral patellar dislocation results in bone contusion at the medial patella and along the lateral aspect of the lateral femoral condyle. The patellar usually relocates, and the typical bone contusions are the key MRI features to diagnose transient lateral patellar dislocation (Fig. 7). Osteochondral fractures are common in acute or recurrent transient lateral patellar dislocation, seen in up to $70 \%$ of cases. These are most often found at the inferomedial patella or the lateral femoral condyle $[49,61,62]$.

\section{$\mathrm{CT}$ role on evaluating patellar maltracking}

For CT evaluation of the patellofemoral joint, patients are positioned supine, with mild external rotation of up to $15^{\circ}$ with padding as needed to facilitate a relaxed state of the quadriceps musculature. Both knees are scanned simultaneously. The contralateral side may serve as an internal control or may also have anatomic factors predisposing to maltracking. This protocol can help in evaluating for osseous integrity, morphology, and patellofemoral alignment [63] (Fig. 8). However, patellofemoral tracking is a dynamic process with the spatial relationship between the articular surfaces varying depending on the position of the knee joint [57, 64]. In fact, most patellar maltracking occurs between extension and the first $30^{\circ}$ of flexion. Thus, to assess for maltracking specifically, a multi-stage $\mathrm{CT}$ with a variable number of repeated acquisitions at variable degrees of flexion can also be performed [57]. At $0^{\circ}$ extension, the patellar may lie completely above the level of the trochlea, without direct apposition between the two articular surfaces. However, the patella starts to engage with the trochlea by $30^{\circ}$ and is typically completely engaged by $45^{\circ}$. At less than $30^{\circ}$ of flexion, asymptomatic knees may demonstrate physiologic patellar tilt or subluxation. In addition, symptomatic knees may demonstrate normal engagement between the patella and trochlea beyond $30^{\circ}$ of flexion. Thus, imaging at positions both less than and greater than $30^{\circ}$ can be used to avoid missing maltracking that might be captured at only certain degrees of flexion [64].

Advantages of CT over plain radiography include its cross-sectional capability and ability to generate multiplanar reformations. This allows for greater detailed evaluation of the patellar and trochlear morphology, patellofemoral relationship, and status of the joint. Advantages of CT over MRI include the reduced cost, larger gantry diameter allowing to fit larger patients, faster acquisition with less potential for claustrophobia, fewer absolute and relative contraindications related to implanted devices, and better cortical bone definition. It is therefore helpful in surgical planning. Disadvantages of CT compared to MRI include the use of ionizing radiation, which reduced soft tissue contrast resulting in limited evaluation of the cartilage, tendons, ligaments, muscles, and internal structures of the knee [64]. 


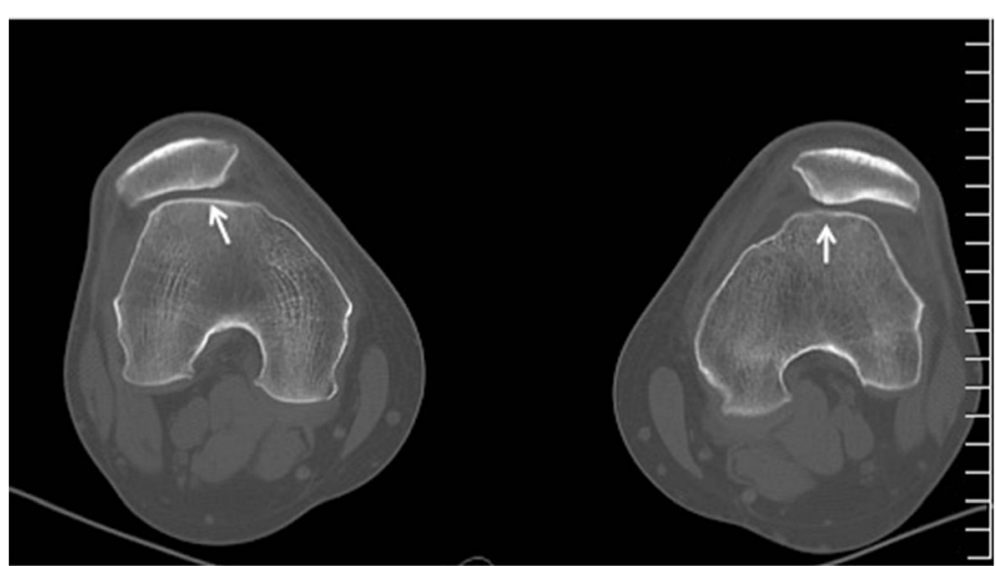

Fig. $8 \mathrm{CT}$ of both knees in $20^{\circ}$ flexion demonstrating bilateral shallow trochlear groove (arrows) compatible with dysplasia and bilateral lateral patellar subluxation and lateral tilt. The two features are associated with patellar maltracking

Features that may predispose to patellar dislocation and/or patellar maltracking and can be evaluated with CT include patellar and trochlear morphology and the alignment between the two structures. These morphological risk factors can be assessed using methods similar to those on MRI as detailed in the prior sections of this article. In acute patellar dislocation, CT may demonstrate osseous impaction or fractures of the medial margin of the patella (with or without involvement of the articular surface) and/or the lateral surface of the lateral femoral condyle and intraarticular fragments. Soft tissue changes may include effusion, thickening or disruption of the MPFL, and retinacular complex and regional edema.

\section{Synopsis of treatment strategies}

The goal of patellar instability treatment is to achieve a stable, functional, and pain-free knee and ultimately to halt or slow the development of osteoarthritis. It can be divided into nonoperative and operative management. The literature in this field has been extremely heterogeneous, and this has made clinical guidelines difficult to produce. A 2015 Cochrane Review concluded that there is no significant increase in functional scores between nonoperative and operative management; however, surgical management does result in a significantly lower risk of recurrent dislocation at the cost of surgical complications [19]. Therefore, the management of patellar maltracking remains controversial and decisions need to be made on an individual patient basis with surgical management being reserved for those patients with documented recurrent lateral patellar instability.

\section{Non-operative management}

For first-time dislocators without intra-articular loose bodies or chondral injury, a trial of nonoperative therapy is indicated. This treatment generally consists of the use of anti-inflammatory medications, a short period of immobilization (3-6 weeks) followed by a progressive physiotherapy regimen with focus on range of motion, closed chain exercises, and vastus medialis obliquus strengthening [16, 65-70]. Adjunctive treatments such as knee aspiration and patellar stabilizing braces have been proposed to decrease swelling and enable early range of motion; however, there is no conclusive evidence for their use [71].

Despite non-operative management, recurrent patellar instability occurs in between 15 and $45 \%$ of patients [17-20]. Additionally, return to sport can be as low as $45 \%$, leaving many patients searching for further management options [12].

\section{Surgical management}

Surgical management of patellar instability should be guided on an individual patient basis depending on history, physical examination, and radiologic findings as outlined above. Over 100 different procedures have been described for the treatment of patellar instability, and this reflects the various causes for instability and lack of current gold standard $[66,69,72]$. The most accepted indication for surgical management of patellar instability is the presence of a large displaced osteochondral fracture or loose body.

Surgical management procedures can broadly be categorized as soft tissue procedures (lateral release, medial imbrication, and MPFL repair or reconstruction) and bony procedures (tibial tubercle transfer procedures and trochleoplasty).

Soft tissue procedures are designed to repair or tighten stretched and injured soft tissues contributing to patellar stability. They are best indicated in isolation in the setting of recurrent instability with minimal underlying 


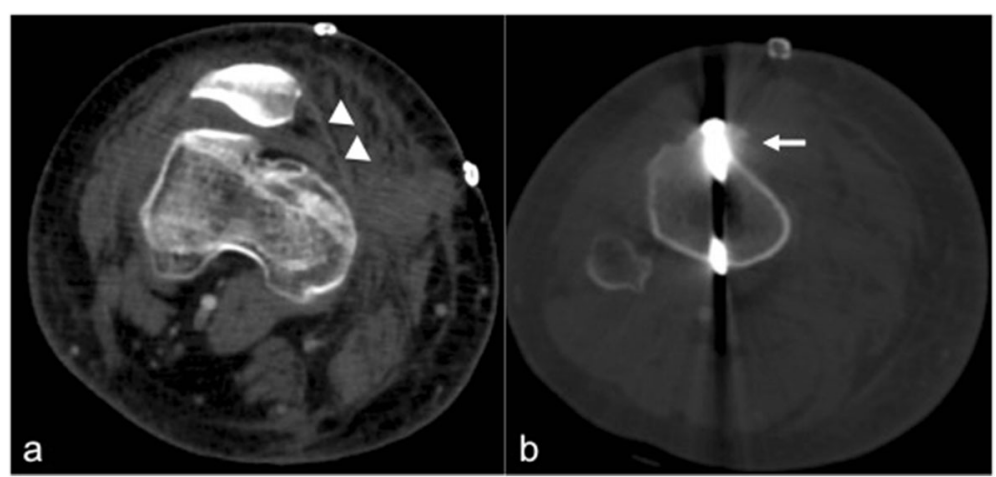

Fig. 9 Knee CT images in the early post-operative period in a 19-year-old male with history of patellar maltracking. a Axial CT image demonstrating MPFL reconstruction (arrow heads). b Axial CT image showing tibial tuberosity transfer surgery with screw placement (arrow)

osseous malalignment (normal TT-TG, minimal trochlear dysplasia). Lateral release and medial imbrication on their own are generally insufficient, but can be used to augment an MPFL repair or reconstruction or, if there is osseous misalignment, used in conjunction with a bony procedure particularly if there is recurrent instability or demonstrable lateral patellar tilt [73-78]. Reconstruction of the MPFL has recently become an increasingly popular procedure for recurrent lateral patellar instability. The technique has been refined, and a better understanding of the anatomical features of both the origin and insertion of the ligament onto the patella has made the operation more reproducible (Fig. 9). Despite this, there remains considerable variation in surgical technique including graft choice, position, and tension making the literature difficult to compare $[8,15,79-86]$.

In the setting of osseous patellar malalignment, an osseous procedure such as tibial tubercle transfer osteotomy can be performed (Fig. 9). Several osteotomies have been described including the medializing Elmslie-Trillat procedure, the anteromedializing Fulkerson osteotomy, and distalization osteotomy [87]. By altering the insertion point of the patellar tendon, these procedures affect the timing and position of patellar engagement in the trochlea and have the ability to biomechanically offload damaged distal articular cartilage, thereby reducing pain and increasing stability simultaneously. It should be noted that these procedures are mostly contraindicated in the patient with open physes due to growth arrest of the tibial tubercle apophysis.

Lastly, a sulcus-deepening procedure known as trochleoplasty may be indicated in the patient with significant trochlear dysplasia and recurrent instability. This procedure involves removal of cancellous bone beneath the trochlea followed by fixation of the articular surface $[88,89]$.

\section{Conclusion}

Patellar maltracking is a disorder that often affects the young active individuals. Early detection particularly in the stage preceding the development of significant cartilaginous loss and osteoarthritis is critical. Imaging plays a vital role in detecting not only the secondary damage but also subtle early features that can raise the suspicion for the presence of this entity. This can provide a road map of developing a treatment strategy that would be primarily aimed at stabilizing the patellofemoral joint and halt the progression of cartilage loss.

\section{Abbreviations}

AP: Anteroposterior; CT: Computed tomography; MPFL: Medial patellofemoral ligament; MRI: Magnetic resonance imaging; TT-TG: Tibial tubercle-trochlear groove

\section{Acknowledgements}

The authors would like to sincerely thank Cheryl Kreviazuk for all her efforts in the submission process.

\section{Authors' contributions}

All authors (ZJ, PJ, KSR, MLS, GD) contributed to the study design, drafted the work, and revised it critically for final submission. All authors read and approved the final manuscript.

\section{Funding}

We confirm that this article is not under consideration for publication elsewhere and that the authors did not receive any specific grants from funding agencies in the public, commercial, or not-for-profit sectors.

\section{Availability of data and materials}

Not applicable

Ethics approval and consent to participate Not applicable

\section{Consent for publication}

Not applicable

\section{Competing interests}

The authors declare that they have no competing interests.

\section{Author details}

'Department of Medical Imaging, The Ottawa Hospital, 501 Smyth Road, Ottawa, ON K1H 8L6, Canada. 'Faculty of Medicine, University of Ottawa, 451 Smyth Road, Ottawa, ON K1H 8M5, Canada. ${ }^{3}$ Division of Orthopaedic Surgery, The Ottawa Hospital, 501 Smyth Road, Ottawa, ON K1H 8L6, Canada. 
Received: 24 January 2019 Accepted: 21 May 2019

Published online: 14 June 2019

\section{References}

1. Post WR, Teitge R, Amis A (2002) Patellofemoral malalignment: looking beyond the viewbox. Clin Sports Med 21:521-546 x

2. Fithian DC, Paxton EW, Stone ML et al (2004) Epidemiology and natural history of acute patellar dislocation. Am J Sports Med 32:1114-1121

3. Tsavalas N, Katonis P, Karantanas AH (2012) Knee joint anterior malalignment and patellofemoral osteoarthritis: an MRI study. Eur Radiol 22: 418-428

4. Sonin AH, Pensy RA, Mulligan ME, Hatem S (2002) Grading articular cartilage of the knee using fast spin-echo proton density-weighted MR imaging without fat suppression. AJR Am J Roentgenol 179:1159-1166

5. Zhang GY, Zheng L, Ding HY, Li EM, Sun BS, Shi H (2015) Evaluation of medial patellofemoral ligament tears after acute lateral patellar dislocation: comparison of high-frequency ultrasound and MR. Eur Radiol 25:274-281

6. Tecklenburg K, Dejour D, Hoser C, Fink C (2006) Bony and cartilaginous anatomy of the patellofemoral joint. Knee Surg Sports Traumatol ArthrosC $14: 235-240$

7. Nakagawa S, Kadoya Y, Kobayashi A, Tatsumi I, Nishida N, Yamano Y (2003) Kinematics of the patella in deep flexion. Analysis with magnetic resonance imaging. J Bone Joint Surg Am 85-A:1238-1242

8. Amis AA, Firer P, Mountney J, Senavongse W, Thomas NP (2003) Anatomy and biomechanics of the medial patellofemoral ligament. Knee 10:215-220

9. Terry GC, Hughston JC, Norwood LA (1986) The anatomy of the iliopatellar band and iliotibial tract. Am J Sports Med 14:39-45

10. Ward SR, Powers CM (2004) The influence of patella alta on patellofemoral joint stress during normal and fast walking. Clin Biomech (Bristol, Avon) 19: 1040-1047

11. Horton MG, Hall TL (1989) Quadriceps femoris muscle angle: normal values and relationships with gender and selected skeletal measures. Phys Ther 69: 897-901

12. Atkin DM, Fithian DC, Marangi KS, Stone ML, Dobson BE, Mendelsohn C (2000) Characteristics of patients with primary acute lateral patellar dislocation and their recovery within the first 6 months of injury. Am J Sports Med 28:472-479

13. Lewallen L, Mclntosh A, Dahm D (2015) First-time patellofemoral dislocation: risk factors for recurrent instability. J Knee Surg 28:303-309

14. Hevesi M, Heidenreich MJ, Camp CL et al (2019) The recurrent instability of the patella score: a statistically based model for prediction of long-term recurrence risk after first-time dislocation. Arthroscopy 35:537-543

15. Mountney J, Senavongse W, Amis AA, Thomas NP (2005) Tensile strength of the medial patellofemoral ligament before and after repair or reconstruction. J Bone Joint Surg Br 87:36-40

16. Wechter J, Macalena J, Arendt EA (1994) Lateral patella dislocations: history, physical exam, and imaging. In: West RV, Colvin AC (eds) The patellofemoral joint in the athlete. Springer, New York, pp 15-29

17. Cash JD, Hughston JC (1988) Treatment of acute patellar dislocation. Am J Sports Med 16:244-249

18. Hawkins RJ, Bell RH, Anisette G (1986) Acute patellar dislocations. The natural history. Am J Sports Med 14:117-120

19. Smith TO, Donell S, Song F, Hing CB (2015) Surgical versus non-surgical interventions for treating patellar dislocation. Cochrane Database Syst Rev 2: CD008106

20. Woo R, Busch MT (1998) Management of patellar instability in children. Oper Tech Sports Med 6:247-258

21. Dejour H, Walch G, Nove-Josserand L, Guier C (1994) Factors of patellar instability: an anatomic radiographic study. Knee Surg Sports Traumatol Arthrosc 2:19-26

22. Jaquith BP, Parikh SN (2017) Predictors of recurrent patellar instability in children and adolescents after first-time dislocation. J Pediatr Orthop 37: 484-490

23. Parikh SN, Lykissas MG, Gkiatas I (2018) Predicting risk of recurrent patellar dislocation. Curr Rev Musculoskelet Med 11:253-260

24. Pfirrmann CW, Zanetti M, Romero J, Hodler J (2000) Femoral trochlear dysplasia: MR findings. Radiology 216:858-864

25. Biedert RM, Tscholl PM (2017) Patella alta: a comprehensive review of current knowledge. Am J Orthop (Belle Mead NJ) 46:290-300

26. Dejour H, Walch G, Neyret P, Adeleine P (1990) Dysplasia of the femoral trochlea. Rev Chir Orthop Reparatrice Appar Mot 76:45-54
27. Diederichs $G$, Issever AS, Scheffler S (2010) MR imaging of patellar instability: injury patterns and assessment of risk factors. Radiographics 30:961-981

28. Tscholl PM, Wanivenhaus F, Fucentese SF (2017) Conventional radiographs and magnetic resonance imaging for the analysis of trochlear dysplasia: the influence of selected levels on magnetic resonance imaging. Am J Sports Med 45:1059-1065

29. Brossmann J, Muhle C, Schröder C et al (1993) Patellar tracking patterns during active and passive knee extension: evaluation with motion-triggered cine MR imaging. Radiology 187:205-212

30. Jibri Z, Martin D, Mansour R, Kamath S (2012) The association of infrapatellar fat pad oedema with patellar maltracking: a case-control study. Skeletal Radiol 41:925-931

31. Wittstein JR, Bartlett EC, Easterbrook J, Byrd JC (2006) Magnetic resonance imaging evaluation of patellofemoral malalignment. Arthroscopy 22:643-649

32. Carrillon Y, Abidi H, Dejour D, Fantino O, Moyen B, Tran-Minh VA (2000) Patellar instability: assessment on MR images by measuring the lateral trochlear inclination-initial experience. Radiology 216:582-585

33. Ward SR, Terk MR, Powers CM (2007) Patella alta: association with patellofemoral alignment and changes in contact area during weightbearing. J Bone Joint Surg Am 89:1749-1755

34. Miller TT, Staron RB, Feldman F (1996) Patellar height on sagittal MR imaging of the knee. AJR Am J Roentgenol 167:339-341

35. Caton JH, Dejour D (2010) Tibial tubercle osteotomy in patello-femoral instability and in patellar height abnormality. Int Orthop 34:305-309

36. Biedert RM, Albrecht S (2006) The patellotrochlear index: a new index for assessing patellar height. Knee Surg Sports Traumatol Arthrosc 14:707-712

37. Ahmad M, Janardhan S, Amerasekera S, Nightingale P, Ashraf T, Choudhary S (2019) Reliability of patellotrochlear index in patellar height assessment on MRIcorrection for variation due to change in knee flexion. Skeletal Radiol 48:387-393

38. Schoettle PB, Zanetti M, Seifert B, Pfirrmann CW, Fucentese SF, Romero J (2006) The tibial tuberosity-trochlear groove distance; a comparative study between CT and MRI scanning. Knee 13:26-31

39. McNally EG (2001) Imaging assessment of anterior knee pain and patellar maltracking. Skeletal Radiol 30:484-495

40. Tsujimoto K, Kurosaka M, Yoshiya S, Mizuno K (2000) Radiographic and computed tomographic analysis of the position of the tibial tubercle in recurrent dislocation and subluxation of the patella. Am J Knee Surg 13:83-88

41. Izadpanah K, Weitzel E, Vicari M et al (2014) Influence of knee flexion angle and weight bearing on the Tibial Tuberosity-Trochlear Groove (TTTG) distance for evaluation of patellofemoral alignment. Knee Surg Sports Traumatol Arthrosc 22:2655-2661

42. Seitlinger G, Scheurecker G, Högler R, Labey L, Innocenti B, Hofmann S (2012) Tibial tubercle-posterior cruciate ligament distance: a new measurement to define the position of the tibial tubercle in patients with patellar dislocation. Am J Sports Med 40:1119-1125

43. Boutris N, Delgado DA, Labis JS, McCulloch PC, Lintner DM, Harris JD (2018) Current evidence advocates use of a new pathologic tibial tubercleposterior cruciate ligament distance threshold in patients with patellar instability. Knee Surg Sports Traumatol Arthrosc 26:2733-2742

44. Hingelbaum S, Best R, Huth J, Wagner D, Bauer G, Mauch F (2014) The TTTG Index: a new knee size adjusted measure method to determine the TTTG distance. Knee Surg Sports Traumatol Arthrosc 22:2388-2395

45. Escala JS, Mellado JM, Olona M, Giné J, Sauri A, Neyret P (2006) Objective patellar instability: MR-based quantitative assessment of potentially associated anatomical features. Knee Surg Sports Traumatol Arthrosc 14:264-272

46. McNally EG, Ostlere SJ, Pal C, Phillips A, Reid H, Dodd C (2000) Assessment of patellar maltracking using combined static and dynamic MRI. Eur Radiol 10:1051-1055

47. Stefanik JJ, Zumwalt AC, Segal NA, Lynch JA, Powers CM (2013) Association between measures of patella height, morphologic features of the trochlea, and patellofemoral joint alignment: the MOST study. Clin Orthop Relat Res 471:2641-2648

48. Laurin CA, Dussault R, Levesque HP (1979) The tangential x-ray investigation of the patellofemoral joint: $x$-ray technique, diagnostic criteria and their interpretation. Clin Orthop Relat Res 144:16-26

49. Elias DA, White LM, Fithian DC (2002) Acute lateral patellar dislocation at MR imaging: injury patterns of medial patellar soft-tissue restraints and osteochondral injuries of the inferomedial patella. Radiology 225:736-743

50. Kirsch MD, Fitzgerald SW, Friedman H, Rogers LF (1993) Transient lateral patellar dislocation: diagnosis with MR imaging. AJR Am J Roentgenol 161:109-113 
51. Lance E, Deutsch AL, Mink JH (1993) Prior lateral patellar dislocation: MR imaging findings. Radiology 189:905-907

52. Warren LF, Marshall JL (1979) The supporting structures and layers on the medial side of the knee: an anatomical analysis. J Bone Joint Surg Am 61:56-62

53. Jerabek SA, Asnis PD, Bredella MA, Ouellette HA, Poon SK, Gill TJ 4th (2009) Medial patellar ossification after patellar instability: a radiographic finding indicative of prior patella subluxation/dislocation. Skeletal Radiol 38:785-790

54. Campagna R, Pessis E, Biau DJ et al (2012) Is superolateral Hoffa fat pad edema a consequence of impingement between lateral femoral condyle and patellar ligament? Radiology 263:469-474

55. Subhawong TK, Eng J, Carrino JA, Chhabra A (2010) Superolateral Hoffa's fat pad edema: association with patellofemoral maltracking and impingement. AJR Am J Roentgenol 195:1367-1373

56. Jarraya M, Diaz LE, Roemer FW, Arndt WF, Goud AR, Guermazi A (2018) MRI findings consistent with peripatellar fat pad impingement: how much related to patellofemoral maltracking? Magn Reson Med Sci 17:195-202

57. Elias DA, White LM (2004) Imaging of patellofemoral disorders. Clin Radio 59:543-557

58. Ali SA, Helmer R, Terk MR (2010) Analysis of the patellofemoral region on MRI: association of abnormal trochlear morphology with severe cartilage defects. AJR Am J Roentgenol 194:721-727

59. Kalichman L, Zhang Y, Niu J et al (2007) The association between patellar alignment and patellofemoral joint osteoarthritis features--an MRI study. Rheumatology (Oxford) 46:1303-1308

60. Sanders TL, Pareek A, Johnson NR, Stuart MJ, Dahm DL, Krych AJ (2017) Patellofemoral arthritis after lateral patellar dislocation: a matched population-based analysis. Am J Sports Med 45:1012-1017

61. Pedersen ME, DaCambra MP, Jibri Z, Dhillon S, Jen H, Jomha NM (2015) Acute osteochondral fractures in the lower extremities - approach to identification and treatment. Open Orthop J 9:463-474

62. Sanders TG, Paruchuri NB, Zlatkin MB (2006) MRI of osteochondral defects of the lateral femoral condyle: incidence and pattern of injury after transient lateral dislocation of the patella. AJR Am J Roentgenol 187:1332-1337

63. White BJ, Sherman OH (2009) Patellofemoral instability. Bull NYU Hosp Jt Dis 67:22-29

64. Dupuy DE, Hangen DH, Zachazewski JE, Boland AL, Palmer W (1997) Kinematic CT of the patellofemoral joint. AJR Am J Roentgenol 169:211-215

65. Buchner M, Baudendistel B, Sabo D, Schmitt H (2005) Acute traumatic primary patellar dislocation: long-term results comparing conservative and surgical treatment. Clin J Sport Med 15:62-66

66. Colvin AC, West RV (2008) Patellar instability. J Bone Joint Surg Am Volume 90:2751-2762

67. Dixit S, Deu RS (2017) Nonoperative treatment of patellar instability. Sports Med Arthrosc Rev 25:72-77

68. Gillespie H (2012) Update on the management of patellar instability. Curr Sports Med Rep 11:226-231

69. Jain NP, Khan N, Fithian DC (2011) A treatment algorithm for primary patellar dislocations. Sports Health 3:170-174

70. Stensdotter AK, Hodges PW, Mellor R, Sundelin G, Häger-Ross C (2003) Quadriceps activation in closed and in open kinetic chain exercise. Med Sci Sports Exerc 35:2043-2047

71. Steiner T, Parker RD (2009) Patella: subluxation and dislocation. 2. Subluxation and dislocation: recurrent. In: Miller M, Thompson S (ed) DeLee and Drez's orthopaedic sports medicine. Elsevier, Philadelphia

72. Buckens CF, Saris DB (2010) Reconstruction of the medial patellofemoral ligament for treatment of patellofemoral instability: a systematic review. Am J Sports Med 38:181-188

73. Ali S, Bhatti A (2007) Arthroscopic proximal realignment of the patella for recurrent instability: report of a new surgical technique with 1 to 7 years of follow-up. Arthroscopy 23:305-311

74. Kolowich PA, Paulos LE, Rosenberg TD, Farnsworth S (1990) Lateral release of the patella: indications and contraindications. Am J Sports Med 18:359-365

75. Lattermann C, Toth J, Bach BR Jr (2007) The role of lateral retinacular release in the treatment of patellar instability. Sports Med Arthrosc 15:57-60

76. Nam EK, Karzel RP (2005) Mini-open medial reefing and arthroscopic lateral release for the treatment of recurrent patellar dislocation: a medium-term follow-up. Am J Sports Med 33:220-230

77. Nikku R, Nietosvaara Y, Aalto K, Kallio PE (2005) Operative treatment of primary patellar dislocation does not improve medium-term outcome: a $7-$ year follow-up report and risk analysis of 127 randomized patients. Acta Orthop 76:699-704
78. Nikku R, Nietosvaara Y, Kallio PE, Aalto K, Michelsson JE (1997) Operative versus closed treatment of primary dislocation of the patella. Similar 2-year results in 125 randomized patients. Acta Orthop Scand 68:419-423

79. Deie M, Ochi M, Sumen Y, Adachi N, Kobayashi K, Yasumoto M (2005) A long-term follow-up study after medial patellofemoral ligament reconstruction using the transferred semitendinosus tendon for patellar dislocation. Knee Surg Sports Traumatol Arthrosc 13:522-528

80. Farr J, Schepsis AA (2006) Reconstruction of the medial patellofemoral ligament for recurrent patellar instability. J Knee Surg 19:307-316

81. Gonçaives MB, Júnior LH, Soares LF, Gonçaives TJ, Dos Santos RL, Pereira ML (2011) Medial patellofemoral ligament reconstruction to treat recurrent patellar dislocation. Rev Bras Ortop 46:160-164

82. LeGrand AB, Greis PE, Dobbs RE, Burks RT (2007) MPFL reconstruction. Sports Med Arthrosc Rev 15:72-77

83. Mikashima Y, Kimura M, Kobayashi Y, Miyawaki M, Tomatsu T (2006) Clinical results of isolated reconstruction of the medial patellofemoral ligament for recurrent dislocation and subluxation of the patella. Acta Orthop Belg 72:65-71

84. Panagopoulos A, van Niekerk L, Triantafillopoulos IK (2008) MPFL reconstruction for recurrent patella dislocation: a new surgical technique and review of the literature. Int J Sports Med 29:359-365

85. Smith TO, Walker J, Russell N (2007) Outcomes of medial patellofemoral ligament reconstruction for patellar instability: a systematic review. Knee Surg Sports Traumatol Arthrosc 15:1301-1314

86. Tom A, Fulkerson JP (2007) Restoration of native medial patellofemoral ligament support after patella dislocation. Sports Med Arthrosc 15:68-71

87. Longo UG, Rizzello G, Ciuffreda M et al (2016) Elmslie-Trillat, Maquet, Fulkerson, Roux Goldthwait, and other distal realignment procedures for the management of patellar dislocation: systematic review and quantitative synthesis of the literature. Arthroscopy 32:929-943

88. Dejour D, Saggin P (2010) The sulcus deepening trochleoplasty-the Lyon's procedure. Int Orthop 34:311-316

89. Donell ST, Joseph G, Hing CB, Marshall TJ (2006) Modified Dejour trochleoplasty for severe dysplasia: operative technique and early clinical results. Knee 13:266-273

\section{Publisher's Note}

Springer Nature remains neutral with regard to jurisdictional claims in published maps and institutional affiliations.

\section{Submit your manuscript to a SpringerOpen ${ }^{\circ}$ journal and benefit from:}

- Convenient online submission

- Rigorous peer review

- Open access: articles freely available online

- High visibility within the field

- Retaining the copyright to your article

Submit your next manuscript at $>$ springeropen.com 